\title{
Pharmacological knowledge loss among medical students in Ahvaz Jundishapur University of Medical Sciences
}

\author{
Zaeemzadeh ${ }^{1}{ }^{1 a}$, Mard S. A. ${ }^{2 a}$, Khosravi M. ${ }^{1}$, Lajmirnia M. ${ }^{1}$, Sedighi $A .{ }^{1}$
}

\begin{abstract}
Introduction: The knowledge loss, especially in medicine has long been an issue of concern for all health care providers and is poorly investigated throughout the world. In this study we focused on pharmacological knowledge in Ahvaz Jundishapur University of Medical Sciences in Iran.

Methods: 110 volunteer of medical students who had previously passed two partial basic pharmacology exams were retested (an unscheduled retention test) again in three group, which were in the 9th $(n=44)$, 12th $(n=37)$, and 15th $(n=29)$ semester of their education to be evaluated for loss of their pharmacological knowledge.

Results: The average score of partial exams in all three groups was significantly higher than the score of retention test $(12.74 \pm 0.31$ vs. $9.93 \pm 0.44,15.85 \pm 0.35$ vs. $12.57 \pm 0.43$, and $13.31 \pm 0.35$ vs. $11.68 \pm 0.61$ of the students spending 9th, 12th, and 15th semesters, respectively). The amount of knowledge loss was $22 \pm 0.6,20 \pm 0.7$, and $12 \pm 0.75 \%$ of the students spending 9th, 12th, and 15th semesters, respectively. There was a significant decrease in the value of knowledge loss of the students spending the15th semester in comparison to the other groups $(p<0.05)$.
\end{abstract}

Conclusion: These results showed that when the time period between the first exposure to pharmacological education and retention test, is increased, the amount of knowledge loss becomes smaller. The reason may be due to more practical rehearsal of pharmacology in clinical years.

Key words: knowledge loss, medical student, pharmacology, retention

\section{Introduction}

Forgetting the knowledge learnt, has ever been a matter of concern and also an issue of research from a long time ago (Eickenberry, 1923). There is plenty of research in this field on a variety of domains, including: Chemistry (Arzi et al., 1986), Psychology (Ellis et al., 1998), Zoology (Tyler, 1933), Botany (Johnson, 1930) Oral Biology (Watt, 1987), and so on.

\section{Abdolshakour Sedighi ${ }^{1}$ \\ ${ }^{1}$ Dept. of Pharmacology, the School of Medicine, \\ Ahvaz Jundishapur University of Medical Sciences, \\ Ahvaz, \\ Iran.}

${ }^{2}$ Research Institute for Infectious Diseases Of Digestive System, Physiology Research Center (PRC) and Dept. of Physiology, the School of Medicine,

Ahvaz Jundishapur University of Medical Sciences,

Ahvaz,

Iran.

$a=$ they are equally involved; First co-author

\author{
Corresponding author: \\ Narjes Zaeemzadeh, \\ Dept. of Pharmacology, The School of Medicine, \\ Ahvaz Jundishapur University of Medical Sciences, \\ Ahvaz, \\ Iran.
}

Email: zaeemzadeh-n@ajums.ac.ir; alimard77@gmail.com

In medical education this issue becomes more serious on behalf of its criticality. There is a long-standing belief among physicians that basic science knowledge in medical education is going to be highly forgotten when the medical students enter the clinical section of their education (Norman, 2000). Some investigators use the term "disuse atrophy" for what happens to basic science knowledge after years of preclinical exposure (Cole, 1932). Some believe that this event is more severe with special courses like Anatomy (Bethe, 1928) and Biochemistry (Kennedy et al., 1981).

On in the other hand there is some evidence which approve that this belief is not completely true (Ellis et al., 1998). 
The reason maybe the fact that many physicians have not enough confidence about their memory after passing many years of acquiring the basic knowledge (Conway et al., 1991).

Investigations on basic science knowledge retention in medical domains are not common, but there are studies on Biochemistry (Saffran et al., 1981; Blizard et al., 1975; Lazic et al., 2006; Rico et al., 1981; Schwartz, 1981), Anatomy (Blunt \& Blizard, 1975; Sinclair, 1965), Immunology (Marcel, 2006), Physiology, Microbiology, Pathology, Pharmacology (DuBois et al., 1969), Neurosciences (Levine \& Forman, 1973), Biology (Krebs et al., 1997) and Behavioural Sciences (Kennedy et al., 1981; Harrison, 1995) throughout the world.

Custers in 2010 concluded that in medical education, approximately two-third to threefourths of knowledge will be retained after passing one year (Custers, 2010).

This study aimed to evaluate the knowledge retention of Pharmacology in medical students of Ahvaz Jundishapur University of Medical Sciences in Iran to determine if findings are in line with the results of other studies in this domain in the world or not.

\section{Methods}

The study population consisted of 110 volunteers (from 146 medical students in our access, 110 volunteered to cooperate in this study) of medical students at Ahvaz Jundishapur University of Medical Sciences who had previously passed two partial basic pharmacology courses at the 6th and 7th semesters just before the beginning of clinical disciplines at the 8th semester.

A retention test (RT) was held in summer 2014. At that time 44, 37, and 29 volunteers from the sum of 110 were spending the 9th, 12th, and 15 th semester, respectively. The students were not aware of the test in advance.

The RT consisted of 20 multiple choice questions (MCQs) to evaluate the retention of pharmacological knowledge. Each question was followed by 4 answers, only one of which was correct. The questions were selected from a pool of questions previously validated and used in the pre-internship exams. The selected questions covered the topics of pharmacology which had been previously taught to these students according to the university curriculum. The number of questions in each field was as below: drugs affecting autonomic nervous system (3), cardiovascular system (7), respiratory system (1), gastrointestinal tract (1), central nervous system (2), endocrine system (3), antibiotics (2), and chemotherapeutic agents (1). One point was allocated for a correct answer and none for a blank or incorrect answer. The time needed for the test was considered 20 minutes, exactly similar the time considered for their previous partial exams in the pharmacology courses (1 minute for each question). The answers were manually reviewed and scored.

The results of the RT were compared to the average scores of two basic pharmacology partial exams obtained by the same students. In the partial tests, each exam consisted of 50 MCQs with the same characteristics of questions in RT and applied only to the corresponding portion (half of the total topics of basic pharmacology).

\section{Statistical analysis}

The average score of partial exams and the score of RT were expressed as crude score from a maximum of 20 points. Data were analysed using SPSS version 17 and were expressed as mean $\pm S E M$. Paired sample t-test was used for comparing the score of RT and the average score of partial exams. $p<0.05$ was set as significant.

\section{Results}

This study was conducted on a total of 110 medical students of both sexes, 9th semester (female: $61.3 \%$ and male: $38.7 \%$ ), 12th semester (female: $56.7 \%$ and male: $43.3 \%$ ), and 15th semester (female: $72.4 \%$ and male: $27.6 \%$ ), between $23-26$ years of age. All participants were taught basic pharmacology for 1 year, two consecutive semesters (two hours per week).

In this study, retention intervals $(\mathrm{R} I=$ the period of non-use, the time period between the retention test and the initial partial exams in this study) were 1, 2.5, and 4 years in the students who were spending 9th, 12th, and 15th semesters respectively at the time of holding the RT.

The students spending the 9th semester have had a period of one semester (8th) of subinternship (observation of medical practice) after finishing the basic pharmacology courses.

The students spending the 12th semester have had a period of four semesters (8th, 9th, 10th, and 11th) of sub-internship after finishing the basic pharmacology courses. 
The students spending the 15th semester have had a period of five semesters (8th, 9th, 10th, and 11th, 12th) of sub-internship and two semesters (13th, and 14th) of internship (medical practice under supervision) after finishing the basic pharmacology courses. This group is spending the last semester and in fact will be graduating soon.
Figure 1. Shows that the average score of partial exams of all three groups is significantly higher than the score of retention test $(12.74 \pm 0.31$ vs. $9.93 \pm 0.44,15.85 \pm 0.35$ vs. $12.57 \pm 0.43$, and $13.31 \pm 0.35$ vs. $11.68 \pm 0.61$ of the students spending 9th, 12th, and 15th semesters, respectively).

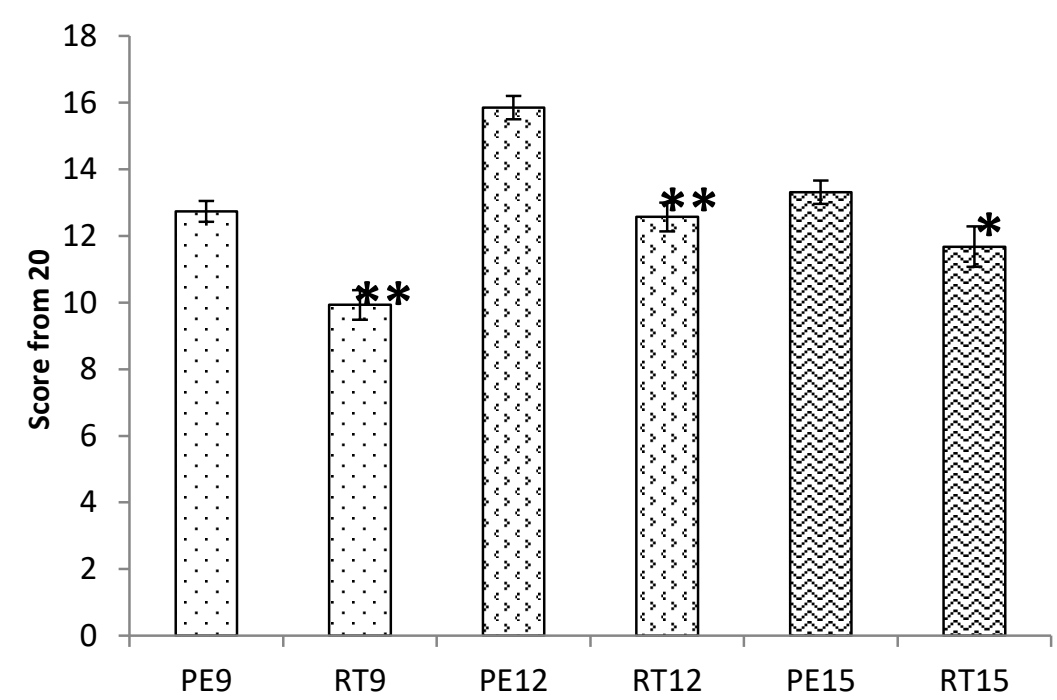

Figure 1: The comparison between the partial exams (PE) and the retention test $(R T)$ scores of medical students spending 9 th, 12 th and 15th semesters. $* P<0.05$ and $* * P<0.01$ as compared with the corresponding partial exams.

Figure 2 compares the amount of knowledge loss (expressed as percentage), of the three groups of students.

The amount of knowledge loss was $22 \pm 0.6 \%$, $20 \pm 0.7 \%$, and $12 \pm 0.75 \%$ of the students spending 9th, 12th, and 15th semesters, respectively. Therefore the amount of retention of knowledge was $78 \%, 80 \%$, and $88 \%$, respectively.
There was no significant difference between the quantities of knowledge loss in the students spending 9th and 12th semesters. But there was a significant decrease in the values of knowledge loss of the students spending 15th semester in comparison to those spending 9th and 12th semesters $(P<0.05)$. 


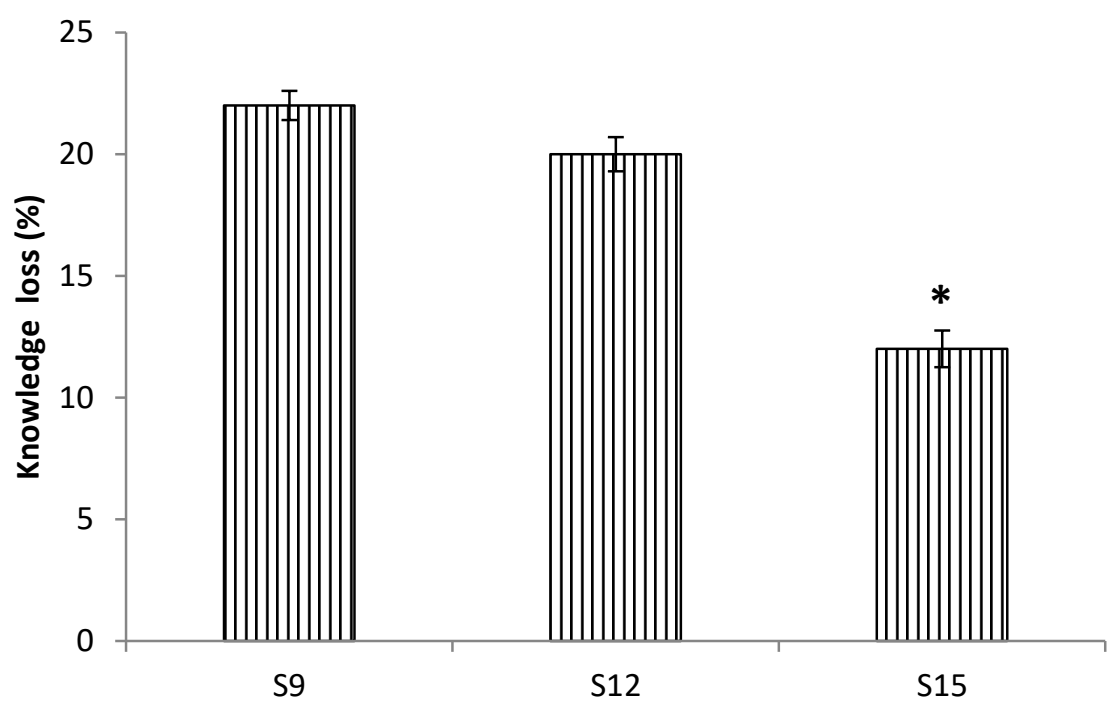

S9: Ninth semester, S12: Twelfth semester, S15: Fifteenth semester. ${ }^{*} \mathrm{P}<0.05$ as compared with S9 and S12

Figure 2: The comparison of the percentage of "knowledge loss" between the three studied groups.

\section{Discussion}

The results showed that passing of time will affect the retention of knowledge any way, as it was seen in all three groups of students who had a retention test score lower than the average of initial partial exams.

In the present study we obtained a knowledge loss values of $22 \%, 20 \%$, and $12 \%$ of the students spending semesters 9, 12, and 15 (RIs $=12,30$, and 48 months, respectively). As the knowledge loss is representative of retention (in a reverse way), the amounts of pharmacological retention were $78 \%, 80 \%$, and $88 \%$, respectively; which are highly acceptable.

We expected that the longer the RI, the lesser the retention of knowledge. Surprisingly our results not only did not show this, but also showed an opposite result; the knowledge loss became quantitative less, when the Rls became longer. It means, the students spending 12th semester had improved retention of knowledge comparing with that of the students in 9th semester, and the students in 15th semester had significantly the highest retention of pharmacological knowledge in compared to the other two groups. These improvements suggest that the exposure of medical students to the clinical disciplines which are started in 8th semester in the Iran medical curriculum can reinforce the prior knowledge of pharmacology. Students in the 9th semester are almost in the beginning of clinical courses in the hospital and involve with patients, but not with drug therapy. In the 12th semester they enter their internship and involve with treatment and drug regimens too. In the 15th semester the medical students are going to be graduated and maybe this is the reason of the lowest knowledge loss (or the highest retention of knowledge) in this group. Bahrick and Weitman believe that incidental short contact with a domain (like Biochemistry) in the $\mathrm{RI}$ has no detectable effect on retention of the knowledge (Bahrick, 1984;Weitman, 1964). This fact cannot be extrapolated to disciplines like Pharmacology, as the exposure of medical students to pharmacological aspects of the illnesses and drug therapy during the clinical years is strong and becomes stronger as they go on. This can impress greatly on the retention of pharmacological knowledge as shown in the present study. 
The research conducted by Kennedy and Saffran showed a retention percentage of pharmacological knowledge higher than $100 \%$ (RIs=24, and 22 months, respectively). In both of these studies the retention exams were performed after passing clinical courses, like the present study, but the difference is the shorter RIs in their studies (22 and 24 months in contrast to 30 and 48 months in the present study). Another reason for this result can be the fact that according to the NOP World Culture Score Index (2014) the USA is among the first thirty countries which have the highest per capita reading (Kennedy et al., 1981; Saffran et al., 1982).

Rodriguez in 2002 also reported a retention percentage higher than $100 \%$ ( $R \mathrm{l}=8$ months) which is higher than what is seen in this study with the similar RI (12 months). Rodriguez states that this improvement is due to presenting a new method in teaching pharmacology which focuses on pharmacologic prototypes (Rodriguez et al., 2002).

Our results ( $R I=12$ months) are in agreement with Herzig's findings ( $\mathrm{Rl}=9$ months) in which it was shown a retention of $80 \%$ in pharmacological knowledge which is similar to $78 \%$ in our study (Herzig et al., 2003).

Only one study, was done by DuBois showed $73 \%$ retention of pharmacological knowledge which is less than the least of what obtained in our study (DuBois et al., 1969). The reason maybe the longer RI (4-6 years) in the DuBois study and the fact that the subjects in this research were the graduates of medical schools not the medical students. This will surely impress on retention of knowledge negatively, as after graduating, the physician involves only with his/her job and studies much less in any domain of medicine.

Swanson et al., in 1996 showed that the performance on basic science disciplines which concerned abnormal processes and treatment can improve over time, whereas performance in normal processes domains usually declines (Swanson et al., 1996). Our results are parallel with this fact, because while the students are advancing in their clinical years and obviously become more expert in clinical issues, the percentage of knowledge loss in pharmacology domain, which is related to abnormal processes and treatment, becomes less.

Overall, in addition to all factors named as the reasons for differences between results in various studies, the retention quantity is related to other factors like teacher quality and student performance which have neither been assessed nor generally is easy to assess in any of the above-mentioned studies, including the present study.

\section{Conclusion}

These results showed that when the time period between the first exposure to pharmacological education and retention test is increased, the amount of knowledge loss becomes lesser. This fact indicates, as the related practical rehearsal in clinical years for pharmacology is more than the other basic sciences in medical education, its retention becomes more and more as time is passing in clinical courses.

\section{References}

Arzi, H.J., Ben-Zvi, R. \& Ganiel, U. (1986) Forgetting versus savings, The many facets of long-term retention, Science Education, 70,2, pp. 171-188.

Bahrick, H.P. (1984) Semantic memory content in permastore: fifty years of memory for Spanish learned in school, Journal of Experimental Psychology: General, 113, 1, pp. 1.

Bethe, A. (1928) Kritische Betrachtungen über den vorklinischen Unterricht, Journal of Molecular Medicine, 7, 31, pp. 1481-1483.

Blizard, P.J., Carmody, J.J. \& Holland, R.A. (1975) Medical students' retention of knowledge of physics and chemistry on entry to a course in physiology, Medical Education, 9, 4, pp. 249-254.

Blunt, M.J. \& Blizard, P.J. (1975) Recall and retrieval of anatomical knowledge, Medical Education, 9, 4, pp. 255-263.

Cole, L. (1932) What is wrong with the medical curriculum ?, The Lancet, 220, 5683, pp. 253-254.

Conway, M.A., Cohen, G. \& Stanhope, N. (1991) On the very long-term retention of knowledge acquired through formal education: Twelve years of cognitive psychology, Journal of Experimental Psychology: General, 120, 4, pp.395.

Custers, E.J. (2010) Long-term retention of basic science knowledge: a review study, Advances In Health Sciences Education , 15,1, pp.109-128.

DuBois, A.B., Nemir Jr,P., Schumacher, C.F. \& Hubbard, J.P. (1969) Graduate medical education in basic sciences, Academic Medicine , 44,11, pp.1035-1043.

Eickenberry, D.H. (1923) Permanence of High School Learning, Journal of Educational Psychology, 14, 8, pp.463.

Ellis, J.A., Semb, G.B. \& Cole, B. (1998) Very longterm memory for information taught in school, Contemporary Educational Psychology, 23, 4, pp.419-433. 
Harrison, A. (1995) Using knowledge decrement to compare medical students' long-term retention of self-study reading and lecture materials, Assessment \& Evaluation in Higher Education, 20,2, pp.149-159.

Herzig, S., Linke, R. M., Marxen, B., Borner, U. \& Antepohl, W. (2003) Long-term follow up of factual knowledge after a single, randomised problembased learning course, BMC Medical Education, 3,1, pp.3.

Johnson, P.O. (1930) The permanence of learning in elementary botany, Journal of Educational Psychology, 21, 1, pp.37.

Kennedy, W.B., Kelley Jr, P.R. \& Saffran, M. (1981) Use of NBME examinations to assess retention of basic science knowledge, Academic Medicine , 56,3, pp. 167-173.

Krebs, R., Guilbert, J.J., Hofer, R. \& Bloch, R. (1997) Retention and forgetting of biological facts and concepts, learnt for the first basic science exam, over a two year period,Advances in Medical Education, pp.162-165.

Lazic, E., Dujmovic, J. \& Hren, D. (2006) Retention of basic sciences knowledge at clinical years of medical curriculum, Croatian Medical Journal, 47,6, pp.882-900.

Levine, H.G. \& Forman, P.M. (1973) A study of retention of knowledge of neurosciences information, Academic Medicine, 48, 9, pp. 867869.

Marcel, F.D. (2006) Knowledge loss of medical students on first year basic science courses at the University of Saskatchewan, BMC Medical Education, 6, 1, pp.5.

Norman, G. (2000) The essential role of basic science in medical education: the perspective from psychology, Clinical and Investigative Medicine, 23, 1, pp. 47-51.
Rico, E., Galindo, J. \& Marset, P. (1981)Remembering biochemistry: a study of the patterns of loss of biochemical knowledge in medical students, Biochemical Education, 9,3, pp.100-102.

Rodriguez, R., Campos-Sepulveda, E., Vidrio, H., Contreras, E. \& Valenzuela, F. (2002) Evaluating knowledge retention of third-year medical students taught with an innovative pharmacology Programme, Academic Medicine, 77,6, pp. 574577.

Saffran, M., Kennedy, W.B. \& Kelley, P.R. (1982) Retention of knowledge of pharmacology by US and Canadian medical students, Trends in Pharmacological Sciences, 3, pp.461-463.

Saffran, M., Kennedy, W.B. \& Kely, P.R. (1981) Use of National Board examinations to estimate retention of biochemistry, Biochemical Education, 9, 3,pp.97-99.

Schwartz, P.L. (1981) Retention of knowledge in clinical biochemistry and the effect of the Keller Plan, Academic Medicine, 56,9, pp. 779-781.

Sinclairl, D.C. (1965) An experiment in the teaching of anatomy, Academic Medicine, 40, 5, pp. 401413.

Swanson, D.B., Case, S.M., Luectht, R.M. \& Dillon, G.F. (1996) Retention of basic science information by fourth-year medical students, Academic Medicine, 71, 10, pp.S80-S82.

Tyler, R.W. (1933) Permanence of learning, Journal of Higher Education, 4, pp. 203-204.

Watt, M.E. (1987) Retention of preclinical knowledge by clinical students, Medical Education, 21, 2, pp. 119-124.

Weitman, M. (1964) A study of long-term retention in medical students, The Journal of Experimental Education,33,1, pp. 87-91. 